\title{
Momentum dependence of the topological susceptibility with overlap fermions
}

\section{Yoshiaki Koma ${ }^{* a}$, Ernst-Michael Ilgenfritz ${ }^{b}$, Karl Koller $^{c}$, Miho Koma $^{a}$, Gerrit Schierholz $^{d}$, Thomas Streuer ${ }^{e}$, Volker Weinberg ${ }^{f}$}

${ }^{a}$ Numazu College of Technology, Numazu, Shizuoka 410-8501, Japan

${ }^{b}$ Institut für Physik, Humboldt-Universität zu Berlin, 12489 Berlin, Germany

${ }^{c}$ Fakultät für Physik, Ludwig-Maximilians-Universität München, 80333 München, Germany

${ }^{d}$ Deutsches Elektronen-Synchrotron DESY, 22603 Hamburg, Germany

${ }^{e}$ Institut für Theoretische Physik, Universität Regensburg, 93040 Regensburg, Germany

${ }^{f}$ Leibniz-Rechenzentrum der Bayerischen Akademie der Wissenschaften, 85748 Garching bei

München, Germany

\begin{abstract}
Knowledge of the derivative of the topological susceptibility at zero momentum is important for assessing the validity of the Witten-Veneziano formula for the $\eta^{\prime}$ mass, and likewise for the resolution of the EMC proton spin problem. We investigate the momentum dependence of the topological susceptibility and its derivative at zero momentum using overlap fermions in quenched lattice QCD simulations. We expose the role of the low-lying Dirac eigenmodes for the topological charge density, and find a negative value for the derivative. While the sign of the derivative is consistent with the QCD sum rule for pure Yang-Mills theory, the absolute value is overestimated if the contribution from higher eigenmodes is ignored.
\end{abstract}

The XXVIII International Symposium on Lattice Filed Theory

June 14-19,2010

Villasimius, Sardinia Italy

* Speaker. 


\section{Introduction}

The derivative at zero momentum of the topological susceptibility $\chi\left(k^{2}\right)$ in momentum space, $\chi^{\prime}(0)$, is important to control the validity of the Witten-Veneziano formula for the $\eta^{\prime}$ mass 11 , 2]. The derivation of this formula presupposes that the momentum dependence of the topological susceptibility $\chi\left(k^{2}\right)$ is moderate from $k^{2}=0$ to $m_{\eta^{\prime}}^{2}$, i.e. $\left|\chi^{\prime} m_{\eta^{\prime}}^{2}\right| \ll \chi$. Moreover, the large- $N_{c}$ limit is implied. The derivative is also helpful to analyze the EMC proton spin problem [3] (see [4] for a review). Therefore it is of great interest to investigate $\chi^{\prime}(0)$ starting from QCD.

Based on various kinds of QCD sum rules, the following estimates of $\chi^{\prime}(0)$ have been reported. For pure gauge (Yang Mills) theory, $\left.\chi^{\prime}(0)\right|_{\mathrm{YM}}=-(7 \pm 3 \mathrm{MeV})^{2}$ [5], and with account of dynamical quarks, $\sqrt{\chi^{\prime}(0)} \approx 23.2 \pm 2.4 \mathrm{MeV}$ [6], $\chi^{\prime}(0)=(2.3 \pm 0.6) \times 10^{-3} \mathrm{GeV}^{2}$ [7], $\sqrt{\chi^{\prime}(0)}=26.4 \pm$ $4.1 \mathrm{MeV}\left(\chi^{\prime} \approx 0.7 \times 10^{-3} \mathrm{GeV}^{2}\right)$ [], and $\chi^{\prime}(0) \approx 1.82 \times 10^{-3} \mathrm{GeV}^{2}$ [].

On the other hand, there are few lattice QCD simulations, which resulted in the following values. In quenched lattice theory, $\left.\chi^{\prime}(0)\right|_{\mathrm{SU}(2)}=-(9.84 \pm 0.91 \mathrm{MeV})^{2}[10],\left.\chi^{\prime}(0)\right|_{\mathrm{SU}(3)}=-(13 \pm$ $16 \mathrm{MeV})^{2}$ [11], and full lattice QCD with staggered fermions, $\sqrt{\chi^{\prime}(0)}=19 \pm 4 \mathrm{MeV}$ [12]. In these simulations, the topological charge density has been defined using the (unimproved) field strength tensor constructed in terms of plaquette variables, and the cooling method has been applied to eliminate the ultraviolet (UV) noise. Here, one may notice that the sign of the $\chi^{\prime}(0)$ estimated by the QCD sum rules is negative in pure Yang-Mills theory while it is positive in full QCD, and the lattice results seem to support this tendency within numerical errors.

In this paper, we are going to use a different approach in order to further investigate the derivative of the topological susceptibility at zero momentum using lattice QCD simulations. We shall use overlap fermions [13, 14] to define the topological charge density [15]. This approach is extremely useful to clarify the topological structure of the QCD vacuum, since it preserves exact chiral symmetry on the lattice [16] and satisfies the index theorem [17]. In this approach, it is also possible to expose the role of the low-lying Dirac eigenmodes for the topological structure of the QCD vacuum [18].

\section{Topological charge density and susceptibility from overlap fermions}

In order to define the topological charge density $q(x)$ on the lattice with the lattice spacing $a$, we employ the massless overlap Dirac operator [13, 14] defined by

$$
D=\frac{\rho}{a}\left(1+\frac{X}{\sqrt{X^{\dagger} X}}\right), \quad X=D_{W}-\frac{\rho}{a},
$$

where $D_{W}$ is the Wilson-Dirac operator. We set $\rho=1.4$, a value identified for the lattices in use as an optimal choice. Details of our implementation are described in [18]. Overlap fermions possess exact chiral symmetry on the lattice [16] and provide $n_{-}+n_{+}$exact zero modes, $D \psi_{n}^{ \pm}=0$, with $n_{-}$ $\left(n_{+}\right)$being the number of modes with negative (positive) chirality: $\gamma_{5} \psi_{n}^{-}=-\psi_{n}^{-}$and $\gamma_{5} \psi_{n}^{+}=+\psi_{n}^{+}$. The index is given by $Q=n_{-}-n_{+}$[17]. The non-zero modes with eigenvalue $\lambda, D \psi_{\lambda}=\lambda \psi_{\lambda}$, occur in complex conjugate pairs $\lambda$ and $\lambda^{*}$ and satisfy $\sum_{x} p_{\lambda 5}(x)=\sum_{x} \operatorname{Tr} \psi_{\lambda}^{\dagger}(x) \gamma_{5} \psi_{\lambda}(x)=0$, where Tr should be regarded as the sum over color and spinor indices. 
Then, the topological charge density $q(x)$ is given by [15]

$$
q(x) \equiv-\operatorname{Tr}\left[\gamma_{5}\left(1-\frac{a}{2} D(x, x)\right)\right]
$$

which satisfies the index theorem with $Q=\sum_{x} q(x) \in \mathbb{Z}$. The contribution of the low-lying Dirac eigenmodes to $q(x)$ can be exposed by applying the eigenmode expansion [19, 20, 21],

$$
q\left(x ; \lambda_{\text {cut }}\right)=-\sum_{|\lambda| \leq \lambda_{\text {cut }}}\left(1-\frac{\lambda}{2}\right) p_{\lambda 5}(x),
$$

where the cut-off $\lambda_{\text {cut }}$ implies a kind of UV filtering, and $p_{\lambda 5}(x)=\psi_{\lambda}^{\dagger}(x) \gamma_{5} \psi_{\lambda}(x)$ is the local chirality of the mode corresponding to the eigenvalue $\lambda$. Note that the UV filtering maintains the index theorem independently of the cut-off such that $Q=\sum_{x} q\left(x ; \lambda_{\text {cut }}\right)$. This is because the index is computed only from the zero modes. Actually, it is observed that all zero modes of a lattice configuration occur with the same chirality. Truncating the expansion at $\lambda_{\text {cut }}$ acts like an UV filter by removing certain short distance fluctuations from the local density $q(x)$.

In the continuum limit, the momentum dependent topological susceptibility is defined as

$$
\chi\left(k^{2}\right)=\int d^{4} x e^{i k x} C_{q}(x),
$$

where $C_{q}(x)=\langle T(q(x) q(0))\rangle$ is the two-point correlation function of the topological charge density $q(x)$. Eq. (2.4) can be expanded with respect to $k^{2}$ and one obtains the expression of the derivative of the topological susceptibility at zero momentum

$$
\chi^{\prime}(0)=\left.\frac{d \chi\left(k^{2}\right)}{d k^{2}}\right|_{k^{2}=0}=-\frac{1}{8} \int d^{4} x x^{2} C_{q}(x) .
$$

The lattice version of Eqs. (2.4) and (2.5) reads

$$
\begin{gathered}
\chi\left(\hat{k}^{2}\right)=\sum_{x \in V} e^{i k x} C_{q}(x), \\
\chi^{\prime}(0)=-\frac{1}{8} \sum_{x \in V} x^{2} C_{q}(x),
\end{gathered}
$$

respectively, where

$$
C_{q}(r)=\frac{1}{V} \sum_{x \in V}\langle q(x+r) q(x)\rangle
$$

As we consider the lattice volume $V=L^{3} T$ and impose periodic boundary conditions in all directions, the momentum is discrete as

$$
\hat{k}_{\mu}=\frac{2}{a} \sin \left(\frac{a k_{\mu}}{2}\right), \quad k_{\mu}=2 \pi\left(\frac{n_{1}}{L}, \frac{n_{2}}{L}, \frac{n_{3}}{L}, \frac{n_{4}}{T}\right),
$$

with $n_{\mu}$ being integer; $\left(n_{1}, n_{2}, n_{3}\right)$ runs from 0 to $(L / a)-1$ and $n_{4}$ from 0 to $(T / a)-1$. 
Table 1: Details of the ensemble used in this study. The Lüscher-Weisz gauge action is used, $\beta$ denotes the inverse coupling squared, $a$ the lattice spacing (determined from the pion decay constant), and $V=L^{3} T$ the lattice volume. $\chi(0)$ is the topological susceptibility.

\begin{tabular}{|c|c|cc|c|l|}
\hline$\beta$ & $a[\mathrm{fm}]$ & $(L / a)^{3}(T / a)$ & $\left(\mathrm{fm}^{4}\right)$ & \# of config. & $\chi(0)\left[\mathrm{GeV}^{4}\right]$ \\
\hline 8.45 & 0.105 & $16^{3} 32$ & $(15.9)$ & 267 & $8.2(7) \times 10^{-4}$ \\
\hline 8.45 & 0.105 & $12^{3} 24$ & $(5.0)$ & 116 & $9.1(13) \times 10^{-4}$ \\
\hline 8.10 & 0.142 & $12^{3} 24$ & $(16.9)$ & 254 & $8.6(8) \times 10^{-4}$ \\
\hline 8.00 & 0.157 & $16^{3} 32$ & $(79.6)$ & 444 & $9.1(6) \times 10^{-4}$ \\
\hline
\end{tabular}

\section{Numerical results}

We used several ensembles of zero-temperature quenched configurations generated by means of the Lüscher-Weisz gauge action [22] (see Table11). This action is suitable for topological studies since dislocations are greatly suppressed.

We plot $\chi\left(\hat{k}^{2}\right)$ as measured at $\beta=8.45$ on the $16^{3} 32$ lattice in Fig 11(left), and as measured at $\beta=8.10$ on the $12^{3} 24$ lattice in Fig. 1 (right), for various cut-off values of the (imaginary part of the) eigenvalue, $\lambda_{\text {cut }}=0.2-0.8 \mathrm{GeV}$. In these analyses, the eigenvalue $\lambda$ of Eq.2.11 is always replaced by $\lambda_{\text {imp }}=(1-a \lambda / 2 \rho)^{-1} \lambda$. These are the eigenvalues of the improved massless overlap operator $D_{\text {imp }}$ [22]. The improvement projects the eigenvalues of $D$ stereographically onto the imaginary axis. We find that the largest value of $\chi\left(\hat{k}^{2}\right)$ is at $\hat{k}^{2}=0$ for each cut-off value of the eigenvalues $\lambda_{\text {cut }}=0.2-0.8 \mathrm{GeV}$ and that the functions $\chi\left(\hat{k}^{2}\right)$ are monotonously decreasing as functions of the momentum for all cut-off values. The qualitative behavior of $\chi\left(\hat{k}^{2}\right)$ does not depend on the lattice spacing.

The result of the $\chi^{\prime}(0)$ according to Eq. 2.7) is plotted in Fig. 2. We do not see a significant volume dependence at $\beta=8.45$ for which two lattice volumes are available. Since the physical volume of the $12^{3} 24$ lattice at $\beta=8.45$ is the smallest among our lattice ensembles (see Table 1 ,
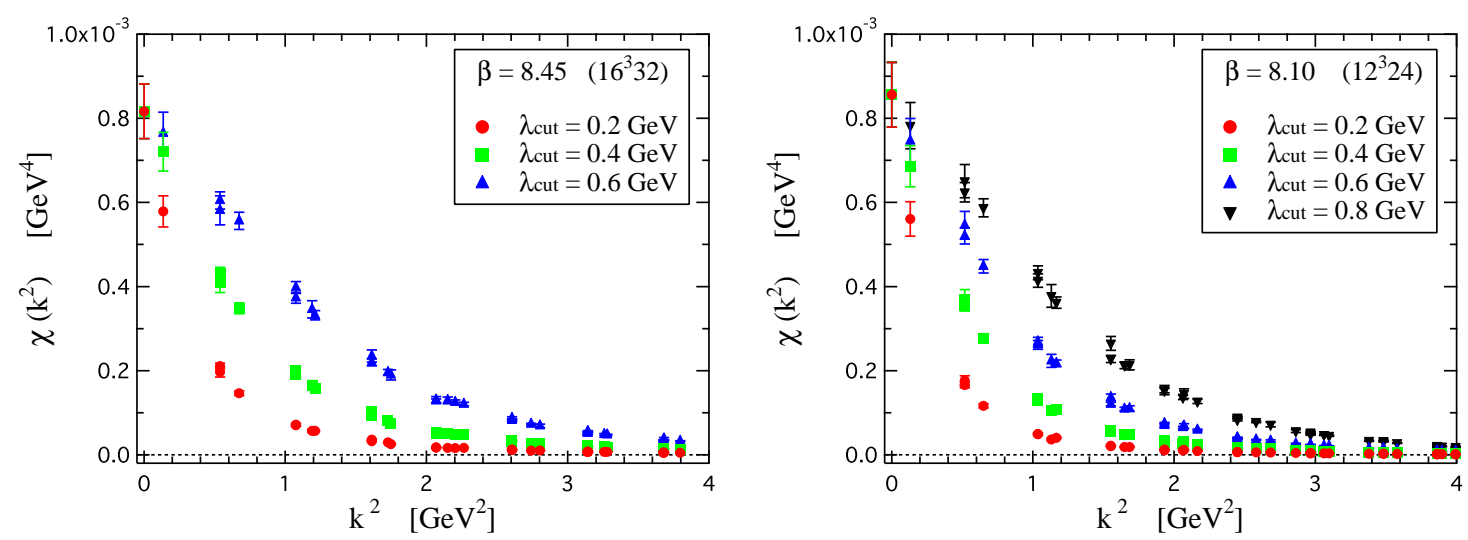

Figure 1: The momentum dependence of the topological susceptibility at $\beta=8.45$ on the $16^{3} 32$ lattice (left). There are two data points at some momenta, which are due to the violation of rotational symmetry, and are expected to converge to one point in the continuum limit. The same as the left figure, but at $\beta=8.10$ on the $12^{3} 24$ lattice (right) . 
we expect that the finite volume effect will not spoil our observation. The physical scales of the cut-off values at different $\beta$ values are chosen approximately corresponding to each other, so as to examine the lattice spacing dependence of $\chi^{\prime}(0)$. In the result we find a reasonable scaling behavior of $\chi^{\prime}(0)$ at corresponding cut-off values $\lambda_{\text {cut }}$, apart from the data of the coarsest lattice at $\beta=8.00$.

In any case, we find an interesting behavior: as the cut-off increases, $\left|\chi^{\prime}(0)\right|$ becomes smaller. In other words, $\left|\chi^{\prime}(0)\right|$ takes on the largest values if only the lowest eigenmodes are included in the fluctuations of the topological density. If one, considering the Witten-Veneziano formula, ignores the necessary condition of $\left|\chi^{\prime}(0) m_{\eta^{\prime}}^{2}\right| \ll \chi(0)$, it seems that the topological susceptibility at zero momentum $\chi(0)$ is the only important parameter for the $\eta^{\prime}$ mass. Since $\chi(0)$ depends only on the (number and chirality of the ) zero modes, one might erroneously conclude that the zero modes are sufficient to describe the topological structure of the QCD vacuum. However, the behavior of $\chi^{\prime}(0)$ indicates that fluctuation of the topological charge density at all scales are necessary to warrant that $\left|\chi^{\prime}(0) m_{\eta^{\prime}}^{2}\right| \ll \chi(0)$ can be satisfied.

For instance, taking a typical value at $\beta=8.10$, we have $\chi^{\prime}(0) \approx-0.001 \mathrm{GeV}^{2}$ $\left(\chi^{\prime}(0) \approx-(32 \mathrm{MeV})^{2}\right)$ with $\chi(0) \approx 8.6 \times$ $10^{-4} \mathrm{GeV}^{4}$, we estimate $\chi(0) /\left|\chi^{\prime}(0)\right| \approx$ $0.86 \mathrm{GeV}^{2}=(930 \mathrm{MeV})^{2}$, which is comparable with $m_{\eta^{\prime}}=958 \mathrm{MeV}$. This in turn means that not only the lowest eigenmodes (not to mention only the zero modes) but also the higher eigenmodes have to play an important role for the topological structure of the $\mathrm{QCD}$ vacuum.

It is then quite intriguing to investigate which value the derivative $\chi^{\prime}(0)$ approaches if more and more contributions from higher eigenmodes (possibly all eigenmodes) are included. We investigate this for the case of $\beta=8.10$ on the $12^{3} 24$ lattice based on the definition of the full topological charge density in Eq. (2.2), which was evaluated without mode expansion. We find the value of the derivative $\chi^{\prime}(0)=-2.4(16) \times$ $10^{-3} \mathrm{GeV}^{2}$. However, we note that the number of configurations used in this analysis is only $53[18]$ and that the topological susceptibility is overestimated for this subsample as $\chi(0)=1.3(3) \times$ $10^{-3} \mathrm{GeV}^{4}$. The limited number of The small number of configurations is dictated by the high cost of evaluating Eq. (2.2) us-

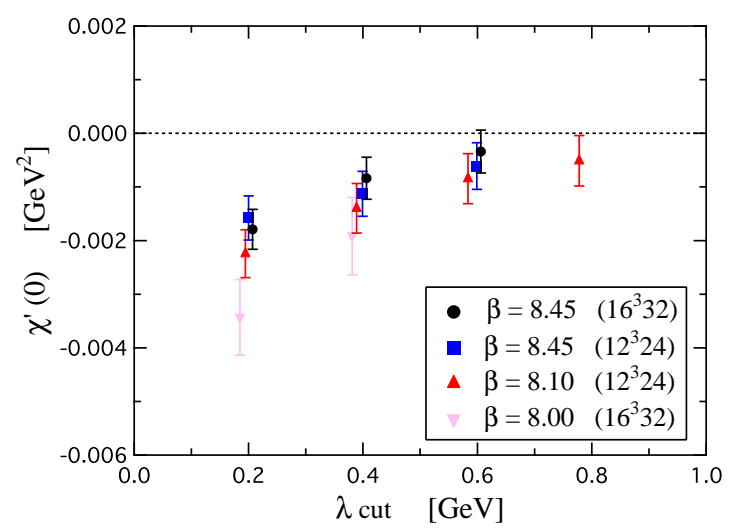

Figure 2: The slope $\chi^{\prime}(0)$ as a function of the cut-off $\lambda_{\text {cut. }}$

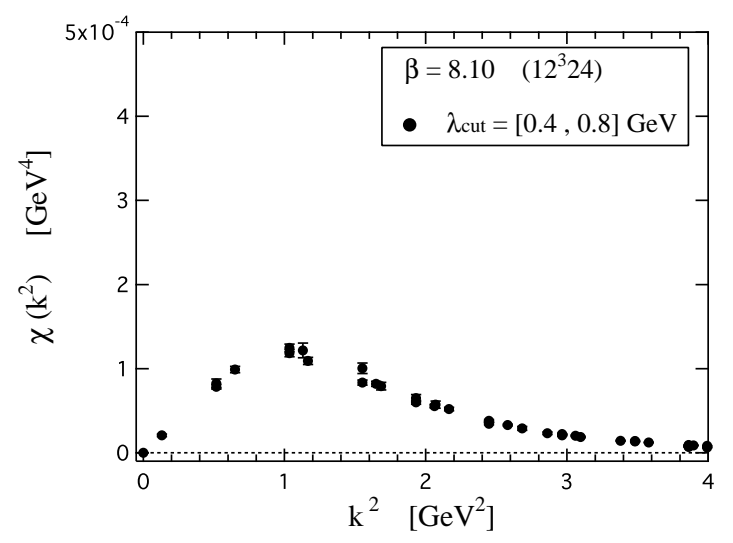

Figure 3: The same as in Fig. 1, but for the ensemble with $\beta=8.10$ on the $12^{3} 24$ lattice, when the zero modes and the lowest eigenmodes are removed from the evaluation of $\chi\left(\hat{k}^{2}\right)$. Only eigenmodes between $0.4 \leq \lambda \leq$ $0.8 \mathrm{GeV}$ are taken into account. 
ing the overlap Dirac operator. Therefore, the value of $\chi^{\prime}(0)$ based on the all-scale topological density (formally $\lambda_{\text {cut }} \rightarrow \infty$ ) cannot be considered as conclusive.

Next we examine the role of the zero and lowest modes for $\chi\left(\hat{k}^{2}\right)$. In Fig. 3, we plot the $\chi\left(\hat{k}^{2}\right)$ for the ensemble with $\beta=8.10$ on the $12^{3} 24$ lattice, where only the eigenmodes between $0.4 \leq \lambda \leq 0.8 \mathrm{GeV}$ are used to define the topological charge density, i.e., the zero modes and very low-lying modes are removed. We find that the slope at $\hat{k}^{2}=0$ turns to be positive. $\chi\left(\hat{k}^{2}\right)$ increases from zero at $\hat{k}^{2}=0$ (due to the subtraction of zero modes from the topological density) to a certain maximum value (near to $\hat{k}^{2} \approx 1 \mathrm{GeV}^{2}$ ) and then decreases.

This leads us to the speculation that - if the zero modes are dynamically suppressed and the relative weight of sectors with smaller $|Q|$ will increase in the average - the sign of the derivative $\chi^{\prime}(0)$ may change from negative to positive. This may be the case in full QCD in the chiral limit.

\section{Summary}

Using the overlap fermion formalism, we have investigated the momentum dependence of the topological susceptibility and its slope at zero momentum, $\chi^{\prime}(0)$. Overlap fermions preserve exact chiral symmetry on the lattice and possess exact zero modes, which allow us to unambiguously compute the index $Q$ of vacuum configurations. We have found that $\chi^{\prime}(0)$ depends on the number of eigenmodes determining the resolution in the definition of the topological charge density. The more the cut-off applied to the eigenvalues of the Dirac operator increases, the more the absolute value of the slope $\left|\chi^{\prime}(0)\right|$ decreases. In other words, $\left|\chi^{\prime}(0)\right|$ with only the lowest-lying eigenmodes included turns out too large to guarantee that the technical assumption underlying the WittenVeneziano formula is fulfilled. From this point of view, fluctuation of the topological charge density at all scales, represented by higher eigenmodes, should also be taken into account. Therefore it is not correct to argue that only zero modes and low-lying eigenmodes are relevant for the topological structure of the QCD vacuum.

As demonstrated in [18], the topological charge density possesses global sign coherent structures, which get increasingly tangled as more and more eigenmodes are included, and the all-scale topological charge density has a lower-dimensional, laminar structure, together with a lumpy structure inside the sign coherent regions. After all, it is found to possess a multifractal structure. This complicated structure of the QCD vacuum is responsible for the behavior of $\chi^{\prime}(0)$.

The numerical calculations have been performed at NIC (Jülich) and HLRN (Berlin) as well as at DESY (Zeuthen). We thank all institutes for support. This work was supported in part by Japan Society for the Promotion of Science (JSPS) and German Research Foundation (DFG), JapanGerman Joint Research Project 2008-2009. Y.K. was partially supported by the Ministry of Education, Science, Sports and Culture, Japan, Grant-in-Aid for Encouragement of Young Scientists (B), No.20740149. M.K. was supported by Japan Society for the Promotion of Science (JSPS), Grant-in-Aid for JSPS Fellows (20 4 40152).

\section{References}

[1] E. Witten, Current algebra theorems for the U(1) Goldstone boson, Nucl. Phys. B156, 269 (1979).

[2] G. Veneziano, U(1) without instantons, Nucl. Phys. B159, 213 (1979). 
[3] European Muon, J. Ashman et al., A measurement of the spin asymmetry and determination of the structure function $g_{1}$ in deep inelastic muon proton scattering, Phys. Lett. B206, 364 (1988).

[4] G.M. Shore, The $U(1)_{A}$ anomaly and QCD phenomenology, Lect. Notes Phys. 737, 235 (2008), hep-ph/0701171.

[5] S. Narison, The slope of the U(1) topological charge from gluonia sum rules and higher order effects on the pseudoscalar meson masses and mixing angles, Phys. Lett. B255, 101 (1991).

[6] S. Narison, G.M. Shore, and G. Veneziano, Target independence of the EMC - SMC effect, Nucl. Phys. B433, 209 (1995), hep-ph/9404277.

[7] B.L. Ioffe and A.G. Oganesian, Proton spin content and QCD topological susceptibility, Phys. Rev. D57, 6590 (1998), hep-ph/9801345.

[8] S. Narison, G.M. Shore, and G. Veneziano, Topological charge screening and the 'proton spin' beyond the chiral limit, Nucl. Phys. B546, 235 (1999), hep-ph/9812333.

[9] J. Pasupathy, J.P. Singh, R.K. Singh, and A. Upadhyay, The derivative of the topological susceptibility at zero momentum and an estimate of eta' mass in the chiral limit, Phys. Lett. B634, 508 (2006), hep-ph/0509260.

[10] G. Briganti, A. Di Giacomo, and H. Panagopoulos, A lattice determination of the slope of the topological susceptibility at $q^{2}=0$, Phys. Lett. B253, 427 (1991).

[11] A. Di Giacomo, E. Meggiolaro, and H. Panagopoulos, A lattice determination of the slope at $q^{2}=0$ of the topological susceptibility in SU(3) Yang-Mills theory, Phys. Lett. B291, 147 (1992).

[12] G. Boyd, B. Alles, M. D'Elia, and A. Di Giacomo, Topology in QCD, (1997), hep-lat/9711025.

[13] H. Neuberger, Exactly massless quarks on the lattice, Phys. Lett. B417, 141 (1998), hep-lat/9707022.

[14] H. Neuberger, More about exactly massless quarks on the lattice, Phys. Lett. B427, 353 (1998), hep-lat/9801031.

[15] F. Niedermayer, Exact chiral symmetry, topological charge and related topics, Nucl. Phys. Proc. Suppl. 73, 105 (1999), hep-lat/9810026.

[16] M. Lüscher, Exact chiral symmetry on the lattice and the Ginsparg-Wilson relation, Phys. Lett. B428, 342 (1998), hep-lat/9802011.

[17] P. Hasenfratz, V. Laliena, and F. Niedermayer, The index theorem in QCD with a finite cut-off, Phys. Lett. B427, 125 (1998), hep-lat/9801021.

[18] E.M. Ilgenfritz et al., Exploring the structure of the quenched QCD vacuum with overlap fermions, Phys. Rev. D76, 034506 (2007), [arXiv:0705.0018 (hep-lat)].

[19] I. Horvath et al., On the local structure of topological charge fluctuations in QCD, Phys. Rev. D67, 011501 (2003), hep-lat/0203027.

[20] Y. Koma et al., Localization properties of the topological charge density and the low lying eigenmodes of overlap fermions, PoS LAT2005, 300 (2006), hep-lat/0509164.

[21] V. Weinberg et al., The QCD vacuum probed by overlap fermions, PoS LAT2006, 078 (2006), hep-lat/0610087.

[22] QCDSF-UKQCD collaboration, D. Galletly et al., Quark spectra and light hadron phenomenology from overlap fermions with improved gauge field action, Nucl. Phys. Proc. Suppl. 129, 453 (2004), hep-lat/0310028. 